\title{
Prospective representation: The effects of varied mapping of sample stimuli to comparison stimuli and differential trial outcomes on pigeons' working memory
}

\author{
ANGELO SANTI \\ Wilfrid Laurier University, Waterloo, Ontario, Canada \\ and \\ WILLIAM A. ROBERTS \\ The University of Western Ontario, London, Ontario, Canada
}

\begin{abstract}
The mapping of sample stimuli onto comparison stimuli and the nature of trial outcomes were factorially manipulated in a delayed matching-to-sample procedure. In the many-to-one condition (MTO), responding to a particular comparison was correct following several sample stimuli, whereas in the one-to-many condition (OTM), responding to several comparison stimuli was correct following a particular sample. Probabilities of reinforcement for correct responding to comparison stimuli were either differential (DO) or nondifferential (NDO). Four groups of pigeons were trained under four combinations of mapping and outcome conditions, MTO-DO, MTO-NDO, OTM-DO, and OTM-NDO. Testing at delay intervals of $1,2,4$, and 8 sec revealed significant effects due to both the mapping variable and the differential outcomes variable. It was argued that the poorer performance obtained in the OTM condition was due to the differential prospective coding requirements placed on reference and working memory by this mapping. In the OTM conditions, a greater number of response codes had to be retrieved from reference memory and multiple response codes may have overburdened working memory, which has a limited capacity.
\end{abstract}

In mastering a task requiring discriminative responses, it is assumed that information concerning which responses to make for particular sequences, which outcomes will follow, and so forth, are organized in a stable knowledge base that transcends the information required to deal with a particular trial. This relatively stable knowledge base is referred to as reference memory (Honig, 1978). Conditional discrimination tasks have been thought to require an additional type of memory, working memory, which is conceived of as short term and limited in capacity. The information held in working memory generally is assumed to be concerned with the particular stimuli that have been presented or are about to be presented on a trial, and is instrumental in determining which response to make in order to obtain reinforcement.

Contemporary theorizing about delayed matching-tosample experiments with animals suggests that informa-

This research was supported by Grant A6378 from the Natural Sciences and Engineering Research Council of Canada to Angelo Santi and by Grant A7894 from the Natural Sciences and Engineering Research Council of Canada to William A. Roberts. These data were reported at the annual meeting of the Psychonomic Society, San Antonio, November 1984. The authors would like to thank Julie Savich, Bill Reason, and Colleen Hanemaayer for their technical assistance. Reprints may be obtained from Angelo Santi, Department of Psychology, Wilfrid Laurier University, Waterloo, Ontario, Canada N2L 3C5. tion derived from the sample stimulus is actively maintained in working memory throughout the delay interval in order to direct responding to comparison stimuli. Further, the information derived from the sample stimulus could be retrospective or prospective in nature (cf. Honig \& Thompson, 1982). Retrospective representation involves the retention in working memory of sample stimulus attributes, whereas the notion of prospective representation holds that the contents of working memory are response instructions about comparison stimuli. The basic distinction is whether working memory contains a representation of "what the animal has seen" or a representation of "what the animal anticipates responding to" (Honig \& Wasserman, 1981; Riley, Cook, \& Lamb, 1981; Roitblat, 1982; Stonebraker, 1981). In favor of the prospective position, the results of several studies have suggested that pigeons performing in delayed matching tasks actively anticipate response decisions (Honig \& Dodd, 1983; Honig \& Wasserman, 1981), trial outcomes (Edwards, Jagielo, Zentall, \& Hogan, 1982; Honig, Matheson \& Dodd, 1984; Peterson, 1984; Peterson \& Trapold, 1980 ), and the duration of memory intervals (Dodd \& Honig, 1981; Wasserman, Grosch, \& Nevin, 1982).

Some experiments have been carried out recently that appear to bear directly on the question of retrospective versus prospective representation in pigeon working 
memory. Roitblat (1980) utilized a choice symbolic delayed matching procedure in which different colors were mapped onto different line tilts. By varying the degree of similarity between hues and the angular orientation of line tilts, Roitblat was able to show that most of the confusion errors made by pigeons were attributable to the similarity of comparison stimuli and not sample stimuli. It was argued from this result that pigeons were retaining information about comparison stimuli and not sample stimuli. In a different experimental design, Stonebraker (1981) superimposed an instructional cue (circle or triangle) on the sample stimulus (red or green field) to signal whether color or line-tilt comparison stimuli would be presented on each trial. When probe tests eventually were presented in which the comparison stimuli were incorrectly cued, there was a significant decrement in performance relative to control trials on which the comparison stimuli were correctly cued. This finding was interpreted as showing that the instructional cue allowed a pigeon to prospectively code its response as the correct member of the appropriate comparison stimulus dimension. In still another study, Grant (1982) utilized a symbolic delayed matching procedure in which three orthogonal sample stimuli (red key, 20 pecks on the sample key, or food delivery) were associated with one comparison stimulus (red key), while three others (green, one peck, and no food) were associated with a second comparison stimulus (green key). It was found that equivalent levels of retention accuracy were obtained when successively presented samples involved either the same physical sample or physically different samples, provided they were associated with the same correct comparison stimulus. Grant concluded that the data were consistent with the prospective view that a single memorial representation of the correct comparison stimulus was encoded when the sample stimuli varied but were associated with a single comparison stimulus. Finally, Kraemer and Roberts (1984) recently examined short-term memory for visual and auditory stimuli in pigeons. By using a symbolic delayed matching procedure, different groups of birds learned to match common visual comparison stimuli to ambient visual or auditory sample stimuli. It was found that several variables, such as delay, intertrial interval and visual or auditory stimulation presented during the delay interval, had common effects on visual and auditory delayed matching. Kraemer and Roberts suggested that these findings were in agreement with the position that pigeons coded both auditory and visual sample stimuli into prospective representations of visual comparison stimuli.

It appears, then, that several lines of evidence can be gathered to support a prospective position. On the other hand, none of these experiments is definitive. Roberts (1982) has suggested ways in which the Roitblat (1980) findings could be explained by a retrospective model, and D'Amato (cited in Honig \& Thompson, 1982) has argued that Stonebraker's (1981) data are consistent with the hypothesis that the birds engaged in retrospective processing of a compound stimulus consisting of the nominal sam- ple stimulus plus the instructional cue. In addition, recent experiments by Spetch and Wilkie (1983) suggest that pigeons may retain retrospective memory traces of sample event durations. It is conceivable that pigeons use both prospective and retrospective coding in different situations. However, one form of coding may be preferred or dominant.

In the experiment presented in this paper, still another approach to the question of prospective versus retrospective processing in pigeon working memory is taken. By varying the number of sample or comparison stimuli used in delayed matching, we were able to create two kinds of mapping conditions, one-to-many (OTM) or many-toone (MTO). In an initial, unreported experiment, pigeons trained on the OTM condition had red or green sample stimuli unpredictably followed by red and green comparison stimuli or vertical and horizontal lines as comparison stimuli; red or vertical line was always correct after a red sample stimulus, and green or horizontal line was always correct after a green sample stimulus. In the MTO condition, separate pigeons were trained on the reverse situation, so that red, green, vertical line, or horizontal line could appear as sample stimuli, but only red and green keys appeared as comparison stimuli, with red correct after either a red or vertical line sample stimulus and green correct after either a green or horizontal line sample stimulus.

Following acquisition of these discriminations, all pigeons were tested at delay intervals of $.5,1,2$, and $4 \mathrm{sec}$. The data obtained were suggestive of better performance in the MTO condition relative to the OTM condition. However, this trend was not statistically significant. The failure to obtain significant effects of the mapping condition in this initial experiment may have been due to the small number of stimuli used. One would expect that differences due to mapping condition would increase as the number of stimuli used was increased. Consequently, in the present experiment, a small white circle and a larger white triangle were added as sample stimuli in the MTO condition and as comparison stimuli in the OTM condition. In the MTO condition, the red comparison was correct after red, vertical line, or circle samples, and the green comparison was correct after green, horizontal line, or triangle samples. In the OTM condition, selection of the red or vertical line or circle comparison stimulus was correct after a red sample, and selection of the green or horizontal line or triangle comparison was correct after a green sample.

Consider the processes necessary for a pigeon to perform the OTM and MTO tasks from retrospective and prospective processing positions. Following initial training to a criterion, an animal presumably has the OTM or MTO mapping relationships stored in reference memory. If retrospective coding is used, a pigeon tested in the OTM condition must remember a trace of the sample stimulus in working memory until the comparison stimuli are presented and then retrieve from reference memory the mapping of the sample stimulus memory onto the re- 
inforced comparison stimulus. Animals tested on the MTO condition must do the same thing; whichever of the six sample stimuli is presented must be held in working memory and then decoded into an appropriate choice when the comparison stimuli are presented. Hence, from the point of view of retrospective memory processing, the OTM and MTO conditions should be of equivalent ease. Such a prediction is clearly not the case from a prospective model. When presented with a sample stimulus in the MTO condition, a pigeon must retrieve from reference memory the appropriate response code corresponding to that sample stimulus and hold that code in working memory until it can be executed at the time comparison stimuli are presented. However, the task becomes more difficult in the OTM condition. In this case, the pigeon is required to retrieve three response codes from reference memory and to hold all of these codes in working memory until one of them can be used to choose between comparison stimuli. There are two ways in which the OTM task may produce more errors than the MTO task. When the sample stimulus is presented, a subject must retrieve only one response code in the MTO task, whereas the subject is required to retrieve three response codes in the OTM task. If retrieval time is restricted, the subject may sometimes retrieve only one code in the OTM condition and thereby reduce its chances of having the correct code to .33 . The greater encoding difficulty of the OTM task over the MTO task may lead to an overall lower level of accuracy in OTM retention curves when compared with MTO retention curves. A second difficulty for the OTM task arises when all three response codes are retrieved. In this case, the memory load is greater in the OTM condition (three response codes) than in the MTO condition (one response code). The limited capacity of working memory then may be overloaded in the OTM condition, leading to more rapid loss of information over the retention interval in the OTM condition than in the MTO condition. Such a differential loss of information would be reflected in a steeper retention curve in the OTM group than in the MTO group.

The above points clearly lead to different predictions from retrospective and prospective models, with the retrospective model predicting little difference between MTO and OTM conditions and the prospective model predicting inferior performance in the OTM condition. A further variable introduced into these experiments may qualify the predictions made from a prospective theory. As a manipulation orthogonal to the mapping variable, pigeons were trained with differential outcomes (DO) or nondifferential outcomes (NDO). In the DO condition, one probability of reinforcement, .2 or 1.0 , was associated with one set of sample and comparison stimuli, and the other probability was associated with the other set of sample and comparison stimuli. In the NDO condition, a single probability of reinforcement, .6 , was in effect with both sets of sample and comparison stimuli. There were, therefore, four groups of pigeons trained under four combinations of mapping and outcome conditions, MTO-DO,
MTO-NDO, OTM-DO, and OTM-NDO. A number of studies have demonstrated enhanced acquisition of discriminations and superior retention in pigeons when sample stimuli are associated with different trial outcomes following correct responding than when outcomes are nondifferential. This DO effect has been demonstrated with qualitatively different reinforcers (Brodigan \& Peterson, 1976; Edwards et al., 1982; Honig et al., 1984; Peterson, Wheeler, \& Armstrong, 1978), reward versus no reward (Peterson, 1984; Peterson \& Trapold, 1980; Peterson, Wheeler \& Trapold, 1980), and differential probability of reward (DeLong \& Wasserman, 1981).

The DO effect most commonly has been attributed to the establishment of differential expectancies that mediate discriminative behavior (Peterson, 1984). With differential probabilities of reinforcement, high and low expectancies of reinforcement will become associated with particular sets of sample and comparison stimuli, and this information will be stored in reference memory. In the OTM-DO condition used in the present research, an animal might learn to expect a high probability of reinforcement when presented with a red sample stimulus and a low probability of reinforcement when presented with a green sample stimulus. Reference memory also would contain the information that, given a high-probability expectancy, pecking a key containing a red field or a vertical line or a circle will yield reinforcement, whereas, given a low-probability expectancy, the appropriate response is to peck a key containing a green field or a horizontal line or a triangle. With the use of such information stored in reference memory, then, during a delay, a pigeon would have to maintain in working memory only an expectancy of high or low probability of reinforcement. The predicted greater difficulty of the OTM problem in comparison with the MTO problem may be greatly reduced when a DO condition is used, because the necessity for remembering three response codes is reduced to remembering one outcome expectancy. When NDO conditions are used, however, the differential encoding and retention requirements of the MTO and OTM problems should continue to exist, and greater difficulty with the OTM condition should be found.

\section{METHOD}

\section{Subjects}

Sixteen White Carneaux pigeons, maintained at $80 \% \pm 20 \mathrm{~g}$ of their ad-lib weight and housed individually with constant access to grit and water, served as subjects. The colony room was lighted from $6 \mathrm{a} . \mathrm{m}$. to $7 \mathrm{p} . \mathrm{m}$., and the subjects usually were run between the hours of 10 a.m. and 2 p.m., 5 days per week. All birds had been experimentally naive prior to the initial experiment briefly described in the introduction.

\section{Apparatus}

Four Coulbourn modular operant test cages (Model \#E10-10), housed individually in isolation cubicles (Model \#E10-20), were used. Each cubicle was equipped with a ventillation fan and baffled air intake and exhaust system. Each test cage was equipped with three, horizontally aligned, clear plastic keys, behind which 
projectors could display stimuli (red or green field, vertical or horizontal white line on a black background, white circle or white triangle on a black background, or a black dot on a white background) onto a frosted rear-projection screen (Coulbourn Model $\#$ E21-18). Directly below the center key was a $5.7 \times 5 \mathrm{~cm}$ opening that provided access to a hopper filled with mixed grain (Coulbourn Model \#E14-10). All experimental events and response measures were arranged and recorded by a microcomputer system located in an adjacent room.

\section{Procedure}

Four groups of pigeons, each containing four birds, were trained to perform 0 -sec delayed matching to sample under different conditions. In the MTO-DO and MTO-NDO groups, the sample stimulus on each trial was red, green, vertical line, horizontal line, circle, or triangle. The comparison stimuli were always red and green side keys. The red comparison stimulus was correct after red, vertical line, or circle sample stimuli, and the green comparison stimulus was correct after green, horizontal line, or triangle sample stimuli. For two of the birds in the MTO-DO group, the probability of reinforcement following a correct response to the red comparison stimulus was 1.0 , whereas the probability for a correct response to the green comparison stimulus was .2. The assignment of probabilities to comparison stimuli was reversed for the other two birds. In the MTO-NDO group, the probability of reinforcement for correct responses was .6 for either comparison stimulus.

In the OTM groups, the sample stimulus was equally often a red or green center key. The comparison stimuli consisted of red and green, or vertical and horizontal lines, or circle and triangle projected on the side keys. Red, vertical line, and circle were correct choices after red samples, and green, horizontal line, and triangle were correct choices after green samples. Two of the birds in the OTM-DO group were reinforced with a probability of 1.0 for correct responses to the red, or vertical line, or circle comparison stimuli and with probability .2 for correct responses to the green, or horizontal line, or triangle comparison stimuli. These probabilities were reversed for the other two pigeons in the OTM-DO group. Birds in the OTMNDO group were reinforced with a probability of .6 for correct responses to all comparison stimuli.

Each trial began with illumination of the center key by the warning stimulus (a black dot on a white background). A peck on the warning stimulus produced one of the six sample stimuli in the MTO condition and one of the two sample stimuli in the OTM condition. The sample stimulus was shown for $5 \mathrm{sec}$ and was followed immediately by presentation of the comparison stimuli on the side keys. The sample and comparison stimuli were used equally often within a session, with the positions of the comparison stimuli on side keys balanced over trials. A correct response to a side key resulted in termination of the comparison stimuli and, on reinforced trials, to 3-sec access to mixed grain. Incorrect responses or correct responses on nonreinforced trials resulted in termination of the comparison stimuli and a 3-sec blackout. Following either reinforcement or the blackout, an intertrial interval of $15 \mathrm{sec}$ was spent in darkness. Each session consisted of 60 trials, or 5 individually randomized blocks of the 12 different trial types in each condition. All birds, except one, were able to maintain performance at or above $85 \%$ correct on these discriminations with a 0 -sec delay procedure. One bird in the OTM-NDO group performed consistently at chance levels and was dropped from the study.

Delay interval (DI) testing was carried out in five blocks of 4 days each. There were four DIs tested, 1, 2, 4, and $8 \mathrm{sec}$, and only one DI duration was tested on a given day. Within each block of 4 days, each DI was tested once, with the sequence of testing varied randomly between birds and between blocks of sessions. Except for the change in DI, all aspects of a test session were identical to those described for the sessions prior to testing. Statistically significant results are reported at the .05 level unless otherwise noted.

\section{RESULTS}

The data obtained during DI testing were collapsed over test sessions for each subject, as a function of whether the trials involved color stimuli alone or a combination of colors and either line tilts or shapes. Accuracy on the trials in which colors alone appeared as sample and comparison stimuli is presented in the left panel of Figure 1; accuracy on mixed trials is presented in the right panel. The data in the left panel provide the cleanest examination of the effects of the mapping variable, since the same colors were used as sample and comparison stimuli in all conditions. In the right panel, comparisons of MTO and OTM conditions are confounded by the use of different stimuli as samples and comparisons.

An analysis of variance on the data in Figure 1 revealed significant effects of outcome condition $[F(1,11)=15.22$, $\mathrm{MSe}=448.88]$, stimulus dimension $[\mathrm{F}(1,11)=16.22$, $\mathrm{MSe}=97.65]$, delay $[\mathrm{F}(3,33)=66.51, \mathrm{MSe}=23.55]$, outcome $\times$ stimulus dimension $\times$ delay $[\mathrm{F}(3,33)=3.25$, $\mathrm{MSe}=10.88]$, and mapping condition $\times$ outcome $\times$ stimulus dimension $\times$ delay $[\mathrm{F}(3,33)=4.07$, MSe $=$ $10.88]$. In order to better understand the four-way interaction, simple effect analyses were performed on the data from color trials and the line-tilt/shape trials. For trials involving shapes and line tilt, the MTO condition was superior to the OTM condition $[F(1,11)=5.28]$, the DO condition was superior to the NDO condition $[F(1,11)=$ 17.05], and accuracy decreased significantly as a function of $\mathrm{DI}[\mathrm{F}(3,33)=44.42]$. No significant interactions were obtained from the line-tilt/shape data. For trials involving colors, significant effects were obtained for outcome $[F(1,11)=9.02]$, delay $[F(3,33)=47.04]$, and mapping condition $\times$ outcome $\times$ delay $[F(3,33)=3.33]$.

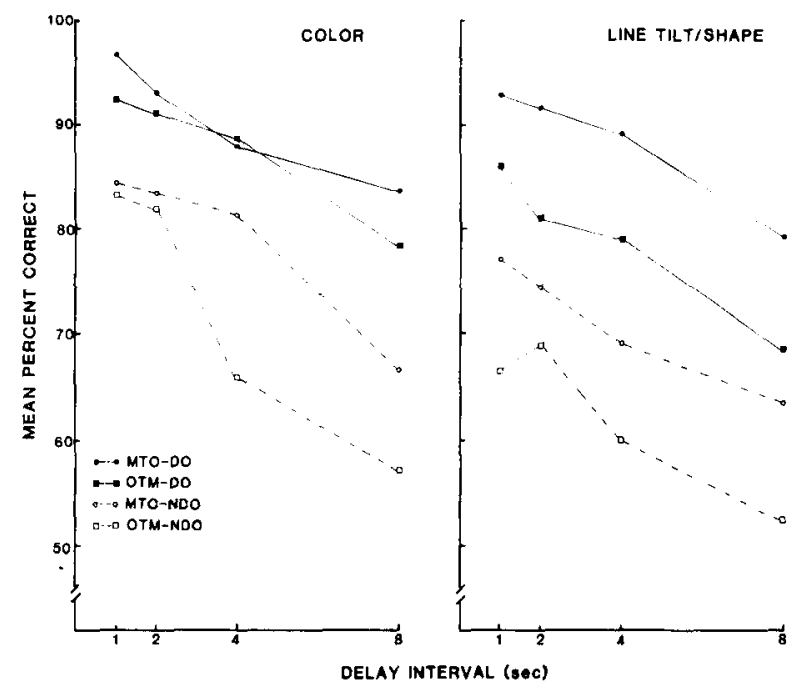

Figure 1. Mean percentage of correct responses during delay interval testing as a function of mapping condition (MTO vs. OTM), trial outcome condition (DO vs. NDO), and stimulus dimension (color vs. line tilt/shape). 
There was no significant difference between the MTODO and OTM-DO groups ( $F<1$ ), although both groups showed declines in accuracy as DI increased $[F(3,33)=$ 14.10]. For the two NDO groups, a significant mapping condition $\times$ delay interaction occurred $[F(3,33)=4.12]$. At DIs of 1 and $2 \mathrm{sec}$, there were no significant differences between the MTO-NDO and OTM-NDO groups (F $<1$ ). The MTO-NDO group was significantly higher than the OTM-NDO group at the 4-sec DI $[F(1,11)=$ $4.80]$ but not at the 8-sec DI $[F(1,11)=1.20]$. Increasing the DI had a greater effect in the OTM-NDO group than in the MTO-NDO group. For the MTO-NDO group, a Newman-Keuls test indicated that performance at an 8-sec DI differed from each of the other DIs, but that no significant differences were found between the 1-, 2-, and 4-sec DIs. In the case of the OTM-NDO group, performance was significantly lower at the 8-sec DI than at each of the other three DIs, and accuracy also was significantly lower at the 4-sec DI than that at the 1- and 2-sec DIs. These analyses support the impression obtained from the left panel of Figure 1 that the OTM-NDO retention curve fell more precipitously between the 2- and 4-sec DIs than did the MTO-NDO retention curve.

\section{DISCUSSION}

The present experiment revealed robust effects of mapping condition and differential outcomes. The effects of these variables are best considered separately for the color trials and the line-tilt/shape trials. In the case of the linetilt/shape trials shown in the right panel of Figure 1, colors were the sample stimuli and line tilts or shapes the comparison stimuli in the OTM conditions, and line tilts or shapes were the sample stimuli and colors the comparison stimuli in the MTO conditions. The overall lower level of accuracy found with trials involving line tilts or shapes than found with trials using only colors may result from greater difficulty in discriminating line tilts or shapes than in discriminating colors. Carter and Eckerman's (1975) analysis of symbolic matching suggested further that matching would be more difficult when line tilts or shapes appeared as sample stimuli and had to be discriminated successively than when they appeared as comparison stimuli and had to be discriminated simultaneously. Their analysis suggests that performance should be higher in our OTM condition, in which line tilts and shapes are used as comparison stimuli, than in our MTO condition, in which line tilts and shapes are used as sample stimuli. The fact that, quite to the contrary from this prediction, the MTO conditions were significantly better than the OTM conditions provides striking evidence for an effect of some factor other than stimulus discriminability. We suggest that this factor is the differential prospective coding requirements of the OTM versus MTO problems. Specifically, the greater difficulty of retrieving three response codes from reference memory in the OTM problem than of retrieving only one response code in the MTO problem would lead to the lower accuracy in the OTM groups.
When color appeared as both sample and comparison stimuli, no difference in ease of discrimination should have existed between the mapping conditions. As seen in the left panel of Figure 1, these trials yielded two findings not seen when line tilts and shapes were used. One was the disappearance of an effect of the mapping variable when outcomes were differential. This finding is in keeping with the suggestion that the differences in the number of prospective codes required by the MTO and OTM groups might be reduced or eliminated when only outcome codes are needed to respond accurately. The second finding is that the difference between the OTM-NDO and MTO-NDO groups appeared as a sharp increase in forgetting for the OTM-NDO group between DIs of 2and 4-sec. This observation supports the notion that the greater memory load required by three codes instead of one could lead to a faster rate of forgetting. The fact that these two phenomena appeared only on color trials and not on trials with line tilt or shape stimuli is puzzling, but both sets of data indicate that the mapping variable had strong effects on delayed matching.

The present study provides further support for the position that memorial representation in pigeons' working memory is primarily prospective in nature. This support derives from the effects obtained as a function of trial outcome conditions, as well as those obtained as a function of mapping conditions. With respect to differential outcomes, the present research replicates the superior memory performance obtained with differential outcomes using probability of reinforcement as the outcome in a choice procedure (Peterson, 1984; Peterson \& Trapold, 1980; Peterson et al., 1980). The DO effect was found under both OTM and MTO mapping conditions. This observation may be interpreted to indicate that pigeons were anticipating trial outcomes in both mapping conditions, rather than retaining sample stimulus attributes (Honig et al., 1984; Honig \& Thompson, 1982). Furthermore, these results suggest that outcome expectations need not mediate responding to a single correct comparison stimulus. In the OTM condition, a DO effect was obtained, and an expectancy of high or low probability of reinforcement in that condition would have to mediate responding to three correct comparison stimuli. The same outcome expectancy may serve as a code for responding to several comparison stimuli.

It was argued in the introduction that the mapping variable manipulated in these experiments should have little effect on performance from the point of view of a retrospective model of working memory, but should be an important factor from the point of view of a prospective model of working memory. Two mechanisms were suggested that should lead to better delayed matching with MTO mapping than with OTM mapping, given that pigeons code sample stimuli into response instructions. First, animals faced with an OTM problem must retrieve more response codes from reference memory than animals faced with a MTO problem; three response codes were necessary in the OTM groups, whereas only one response 
code needed to be retrieved by the MTO groups. Failure to retrieve any one of the response codes in the OTM condition increases the probability of an incorrect response. As a second mechanism, it was suggested that birds burdened with three response instructions in the OTM condition might lose information more rapidly from a limited capacity working memory than would birds required to remember only one instruction in the MTO condition.

Evidence favoring a role for the mapping variable was found in the present research. When colors and line tilts or shapes were used as sample and comparison stimuli, the MTO groups were significantly better than the OTM groups at all delay intervals and under both DO and NDO conditions. Such a finding is in keeping with the difference in retrieval difficulty postulated between MTO and OTM conditions. The consideration of trials on which only colors appeared as sample and comparison stimuli yielded evidence of more rapid forgetting in the OTM condition than in the MTO condition, a finding in line with the notion of an overburdened limited-capacity memory in the OTM condition. However, the mapping effect found with color stimuli was found only under NDO conditions and not under DO conditions. A further theoretical speculation made in the introduction was that DO expectancies might provide an alternative prospective code to response instructions and thus override the otherwise difficult coding requirements of the OTM condition. The interaction of the outcome variable with the mapping variable offers some support for this notion, that a simpler prospective coding system would override a more complicated one.

The results reported obviously do not give unequivocal support to all of the theoretical mechanisms we have suggested. In particular, the types of effects mapping had on performance appeared to be dependent upon the types of sample and comparison stimuli used, in some complicated way that we do not yet fully understand. Nevertheless, we argue that these data provide strong evidence for effects of mapping on delayed matching and that these effects result from the differential prospective coding requirements placed upon reference and working memory by MTO and OTM conditions.

\section{REFERENCES}

Brodigan, D. L., \& Peterson, G. B. (1976). Two-choice conditional discrimination performance in pigeons as a function of reward expectancy, prechoice delay, and domesticity. Animal Learning \& Behavior, 4, 121-124.

Carter, D. E., E Eckerman, D. A. (1975). Symbolic matching by pigeons: Rate of learning complex discriminations predicted from simple discriminations. Science, 187, 662-664.

Delong, R. E., \& Wasserman, E. A. (1981). Effects of differential reinforcement expectancies on successive matching-to-sample performance in pigeons. Joumal of Experimental Psychology: Animal Behavior Processes, 7, 394-412.

DoDD, P. W. D., \& HoNIG, W. K. (1981, June). Effects of signalling the duration of a retention interval in a delayed discrimination. Paper presented at the meeting of the Canadian Psychological Association, Toronto.

Edwards, C. A., Jagielo, J. A., Zentall, T. R., \& Hogan, D. E. (1982). Acquired equivalence and distinctiveness in matching to sample by pigeons: Mediation by reinforcer-specific expectancies. Journal of Experimental Psychology: Animal Behavior Processes, 8, 244-259.

GRANT, D. S. (1982). Prospective versus retrospective coding of samples of stimuli, responses, and reinforcers in delayed matching with pigeons. Learning and Motivation, 13, 265-280.

HoNIG, W. K. (1978). Studies of working memory in the pigeon. In S. H. Hulse, H. Fowler, \& W. K. Honig (Eds.), Cognitive processes in animal behavior (pp. 211-248). Hillsdale, NJ: Erlbaum.

HoniG, W. K. , \& DodD, P. W. D. (1983). Delayed discrimination in the pigeon: The role of within-trial location of conditional cues. Animal Learning \& Behavior, 11, 1-9.

Honig, W. K., Matheson, W. R., \& Dodd, P. W. D. (1984). Outcome expectancies as mediators for discriminative responding. Canadian Journal of Psychology, 38, 196-217.

Honig, W. K., \& Thompson, R. K. R. (1982). Retrospective and prospective processing in animal working memory. In G. H. Bower (Ed.), The psychology of learning and motivation: Advances in research and theory (Vol. 16, pp. 239-283). New York: Academic Press.

Honig, W. K., \& Wasserman, E. A. (1981). Performance of pigeons on delayed simple and conditioned discriminations under equivalent training procedures. Learning and Motivation, 12, 149-170.

Kraemer, P. J., \& RoberTs, W. A. (1984). Short-term memory for visual and auditory stimuli in pigeons. Animal Learning \& Behavior, 12, 275-284.

Peterson, G. B. (1984). How expectancies guide behavior. In H. L. Roitblat, T. G. Bever, \& H. S. Terrace (Eds.), Animal cognition (pp. 135-148). Hillsdale, NJ: Erlbaum.

Peterson, G. B., \& Trapold, M. A. (1980). Effects of altering outcome expectancies on pigeons' delayed conditional discrimination performances. Learning and Motivation, 11, 267-288.

Peterson, G. B., Wheeler, R. L., \& Armstrong, G. D. (1978). Expectancies as mediators in the differential-reward conditional discrimination performance of pigeons. Animal Learning \& Behavior, 6, 279-285.

Peterson, G. B., Wheeler, R. L., \& Trapold, M. A. (1980). Enhancement of pigeons' conditional discrimination performance by expectancies of reinforcement and nonreinforcement. Animal Learning \& Behavior, 8, 22-30.

Riley, D. A., COOK, R. G., \& LAMB, M. R. (1981). A classification and analysis of short-term retention codes in pigeons. In G. H. Bower (Ed.), The psychology of learning and motivation: Advances in research and theory (Vol. 15, pp. 51-79). New York: Academic Press.

ROBERTS, W. A. (1982). Premature closure of controversial issues concerning animal memory representation. Behavioral and Brain Sciences, 5, 384-385.

RoItblat, H. L. (1980). Codes and coding processes in pigeon shortterm memory. Animal Learning \& Behavior, 8, 341-351.

RoITBLAT, H. L. (1982). The meaning of representation in animal memory. Behavioral and Brain Sciences, 5, 353-406.

SPETCH, M. L., \& WILKIE, D. M. (1983). Subjective shortening: A model of pigeons' memory for event duration. Journal of Experimental Psychology: Animal Behavior Processes, 9, 14-30.

STONEBRAKER, T. B. (1981). Retrospective vs. prospective processes in delayed matching-to-sample. Unpublished doctoral dissertation, Michigan State University

Wasserman, E. A., Grosch, J., \& Nevin, J. A. (1982). Effects of signaled retention intervals on pigeon short-term memory. Animal Learning \& Behavior, 10, 330-338.

(Manuscript received August 16, 1984; revision accepted for publication January 23, 1985.) 